\title{
CONTEXTUALIZING ENVIRONMENTAL SUSTAINABILITY IN DESIGN ENGINEERING CURRICULA
}

\author{
Devarajan Ramanujan ${ }^{1 *}$, William Z. Bernstein ${ }^{1}$, Monica Cardella ${ }^{2}, K_{\text {Karthik Ramani }}^{1,3}$ \\ ${ }^{1}$ School of Mechanical Engineering \\ ${ }^{2}$ School of Engineering Education \\ ${ }^{3}$ School of Electrical and Computer Engineering \\ Purdue University, West Lafayette, Indiana 47907
}

\begin{abstract}
Consideration of environmental sustainability is significantly altering the nature of the mechanical design process. This necessitates integration of sustainability related learning content in design engineering curricula. Although various frameworks for teaching sustainable design exist, a survey conducted among practicing student engineers shows the presence of significant knowledge gaps. To this end, we propose a problem-based framework for contextualizing sustainability assessment within design engineering curricula. Our framework makes it possible for embedding sustainability related concepts within traditional engineering courses and promotes discovery learning among students by means of design exploration. We illustrate our approach using a shape synthesis task that integrates environmental assessment into design by constraining the decision space for domain specific variables. Results from a user survey for analyzing the effects of our framework show its ability to promote both awareness and applicability of sustainable design concepts as well as its potential for use in existing engineering curricula.
\end{abstract}

\section{INTRODUCTION}

Educating students on principles of environmental sustainability is one of the primary goals of engineering curricula. The Accreditation Board for Engineering \& Technology (ABET) [1] requires student outcomes to include:

\footnotetext{
*Address all correspondence to this author. Email: dev@ purdue.edu
}

"An ability to design a system, component, or process to meet desired needs within realistic constraints such as economic, environmental, social, political, ethical, health and safety, manufacturability, and sustainability."

Inspite of efforts by educators on disseminating sustainability related research, studies have reported that students lack fundamental understanding and experience related to sustainable development concepts. Azapagic et al.'s [2] survey of more than 3000 students revealed that the overall level of knowledge of sustainable development among participants is not satisfactory. The survey also shows that although students realize the importance of addressing sustainability related issues, there are significant gaps in terms of student knowledge. A related survey of over 4,000 practicing engineers and engineering students conducted by the American Society of Mechanical Engineering and Autodesk shows that $60 \%$ of the respondents expected that their organization's involvement in incorporating sustainable and/or green design specifications would increase in the coming year [3]. Such surveys highlight the need for modifying existing curricula and dissemination methods for sustainable design in order to make it an integral part of the mechanical design process. Within this paper we aim to discuss the following questions related to sustainable design education:

Q1: What are the knowledge gaps in sustainable design present in mechanical engineering students?

Q2: How can we integrate sustainability based learning into design engineering curricula to address some of these gaps? 
Most importantly, we want to look at methods for designing a learning framework that, 1) motivates students to integrate environmental sustainability principles in a design setting and 2) helps students build mental models of the involved design variables by casting this learning as an exploratory process.

We begin the paper by discussing the results of a pilot survey conducted within a graduate-level product design course, illustrating the need for innovative educational techniques in the context of eco-design. Following this, we outline some of the key insights that we are able to infer with regards to teaching concepts of sustainable design. Finally, we discuss the development of a design exploration framework that teaches relationships between environmental sustainability and domain specific variables within mechanical design.

\section{RELATED LITERATURE}

There has been considerable research towards integrating sustainability-based learning into engineering curricula. Pioneering efforts in this context argued for a holistic purview of the subject. Tilbury (1995) [4] states that environmental education for sustainability (EEFS) should focus more sharply on developing closer links between environmental quality, ecology, socioeconomics as well as the political threads which underlie it. The basis for EEFS is the creation of a more holistic outlook on problems, and the formation of an environmental scope. Reorienting education for promoting sustainable development is discussed in [5]. The primary focus therein is the development of an educational system that involves learning the knowledge, skills, perspectives, and values that will guide and motivate people to lead sustainable livelihoods. On similar lines, Ashford (2004) [6] argues that sustainability learning should be interdisciplinary in nature so that it broadens the "design space" for engineers. Inspite of such efforts, surveys conducted by $[2,3]$ have shown the presence of significant knowledge gaps and the inability of students to apply sustainability concepts into engineering practice.

A large portion of recent efforts have been focused towards the development of courses, workshops, games and practical experiences for providing active learning related to sustainability. Brundiers et al. (2010) [7] investigates how experiential learning shape acquisition of key competencies in sustainability. The authors' discover that using a functional and progressive model of real-world learning opportunities helps the above process. The effects of experiential learning games for teaching sustainability is explored in [8]. The authors' group games based on the type of knowledge and learning phase in the Kolb's cycle. This classification forms the basis for three important kinds of games: 1) self-analysis games, 2) system games and 3) communication and collaboration games. The authors' conclude that integrating games into the curricula could prove to be effective in facilitating student learning. Several other researchers $[9,10,11,12]$ have also looked at the effects of simulation games and gamification of real-world tasks to promote awareness of sustainability related concepts. Although such efforts are vital towards promoting and maintaining an active interest in environmental sustainability, a handful of such efforts focus on contextualizing this learning towards engineering practice.

Approaches built on project based learning (PBL) have also been investigated by researchers. Stienemann (2003) [13] discusses sustainability based learning by means of promoting synergies within a university. Using PBL as a framework, the author analyzes successes and difficulties pertaining to the design of projects that promote hands-on sustainability. Ameta et al. (2010) [14] develops a collective learning approach towards teaching sustainability within a systems design course. A majority of project based courses that teach environmental sustainability focus on system level problems, such as water purication and urban infrastructure planning. A notable exception is a PBL approach for teaching Design for Environment (DfE) strategies as discussed in [15]. The authors' develop a critique based module aimed at motivating students to include sustainability considerations into their designs. Results from this study show that integrating a critique based module within an existing design project is an effective method teaching sustainable product design. A survey conducted by the authors' post implementation of this module indicates that students are more likely to apply sustainability principles in engineering practice. A limitation of this approach is that the complexity entailed in such projects limits their applicability to more advanced (graduate level) engineers. Furthermore, in a product design project, explicitly defining the relationships between environmental impact and domain specific design variables becomes a challenge. To bridge this gap, research aimed at integrating sustainability learning in traditional mechanical engineering curriculum is required.

Peet et al. (2004) [16] argues that students find it difficult to integrate sustainable development (SD) into engineering practice unless this learning is incorporated in regular course work. The authors' discuss different techniques related to faculty involvement in teaching SD concepts. They conclude that a semiconsultant approach that involves direct discussions with individual lecturers seems to offer the least resistance for achieving this integration. Approaches for integrating sustainability concepts within mechanical engineering are reviewed in [17]. The authors' conclude that, 1) sustainability education should be fully integrated into design and manufacturing courses and 2) infusing sustainability into engineering curricula is essential for equipping students' with the tools for achieving a sustainable future.

An important aspect in sustainability based education is assessing the type of learning that is involved. Warburton (2003) [18] makes a case for the importance of deep learning in environmental education. The authors' argue that the multidisciplinary and interconnected nature of environmental education necessitates deep learning on behalf of students. Developing curriculum that promotes problem-based learning, a clear 
and concise conceptual framework and promoting personal exploration are viewed as essential components for achieving the former. Learning for sustainability with regards to educational theories on values, attitudes and behaviors is discussed in [19]. The authors' discover that most teaching methods in higher education focus on the cogitative skills of knowledge and understanding rather than the affective outcomes. Potential changes as pointed out by the authors' include: 1) changing assessment outcomes 2) rethinking guidelines for course evaluation and 3 ) designing realistic learning outcomes in the affective domain.

In summary, achieving a synergistic integration of environmental sustainability with design engineering curricula requires a means for embedding sustainability within traditional engineering courses. It is important that the relationship between the two is made explicit and that this integration be made whilst student engineers are learning fundamental concepts of engineering design. Furthermore, ensuring applicability of these concepts requires deep learning on behalf of the students. For this, we propose a possible solution that uses design space exploration for contextualizing sustainability concepts. In this paper, we define deep learning as the cognitive process that produces holistic insight by perceiving underlying meaning, correlations and patterns in disparate topics. It also involves usage of analytical tools to test and redefine gained insight $[18,20]$.

\section{METHODS}

At the beginning of this study, we aimed at understanding current gaps in sustainability related knowledge among design engineering students. For this, we compiled an online survey that was distributed to a class of 40 student engineers. Based on the results from this survey, we developed a framework for better integration of sustainability related concepts within engineering design curricula. The following sections provide details on the online survey, our integration framework and a user study which was conducted to validate our hypotheses.

\subsection{Preliminary User Survey}

To understand the current gaps that persist at the graduatelevel, a pilot survey was conducted within a product design course to assess general awareness of issues related to sustainability. The curriculum of the product design course is designed to expose students to design innovation, market identification and business model development. Students are grouped into project teams and are required to develop an innovative product/service concept as a part of the course requirement. A majority of the students taking this course are full-time working engineers or have 1 to 6 years of industry experience. We received 28 complete responses ( 21 male and 7 female) to the survey with participants ranging from 23 to 35 years of age. The user group encompassed a wide variety of employee designations including design, manufacturing and project engineers, line optimization supervisors and multi-disciplined engineers. A total of 12 participants had previous exposure to environmental sustainability concepts either through work experience or through relevant course work.

To better understand awareness of sustainability related concepts, we compiled a list of topics based on [2] and asked students to rate their self-perceived knowledge in these topics. For this, an online survey was distributed to students before they began developing ideas for their course projects. After the respondents completed their semester long project, another survey was conducted to determine what sort of sustainable and eco-design principles were used within their course projects. Students were asked to submit a detailed report on the life-cycle stages and processes in their design that would significantly contribute to the environmental footprint of their product concept and suggest design changes to mitigate it. Although the product/service ideas generated by the student groups are quite diverse, all of them have aspects that could be designed around the principles that were outlined in the first survey. Significant observations from the online survey and design report are detailed below:

- Participants had a low level of understanding related to ecodesign and sustainability principles. A visual summary of the results obtained from this survey can be seen in Fig.1

- With respect to applicability of previously known sustainability principles, participants had a better understanding of concepts that directly translated to "engineering metrics". For example, principles of material reduction, energy and cost minimization which are well established in traditional engineering curricula were easier to understand and given some consideration within student design projects

- A greater self-perceived knowledge of sustainable development among students did not imply a more comprehensive consideration for eco-design principles within design projects

Reflecting on the results from the preliminary user survey, we can conclude that apart from gaps in awareness, students also face a significant knowledge barrier in terms of applying known principles of sustainability and eco-design to design practice.

\subsection{Framework}

Our framework for teaching sustainability within existing curricula relies on the dependencies of environmental impact as well as design performance on a set of key design variables. In traditional curricula, students solve simplified design problems with the objective of either meeting or optimizing certain objectives. For example, machine design often deals with factors like material selection and shape synthesis to design structural members that can withstand specified loading conditions. Engineering domains such as heat transfer and fluid mechanics also deal with similar problems. Although these problems represent simplifications of the real-world, they teach students about related physical principles and interdependencies among the involved design variables. It is our hypothesis that framing sustainability related 


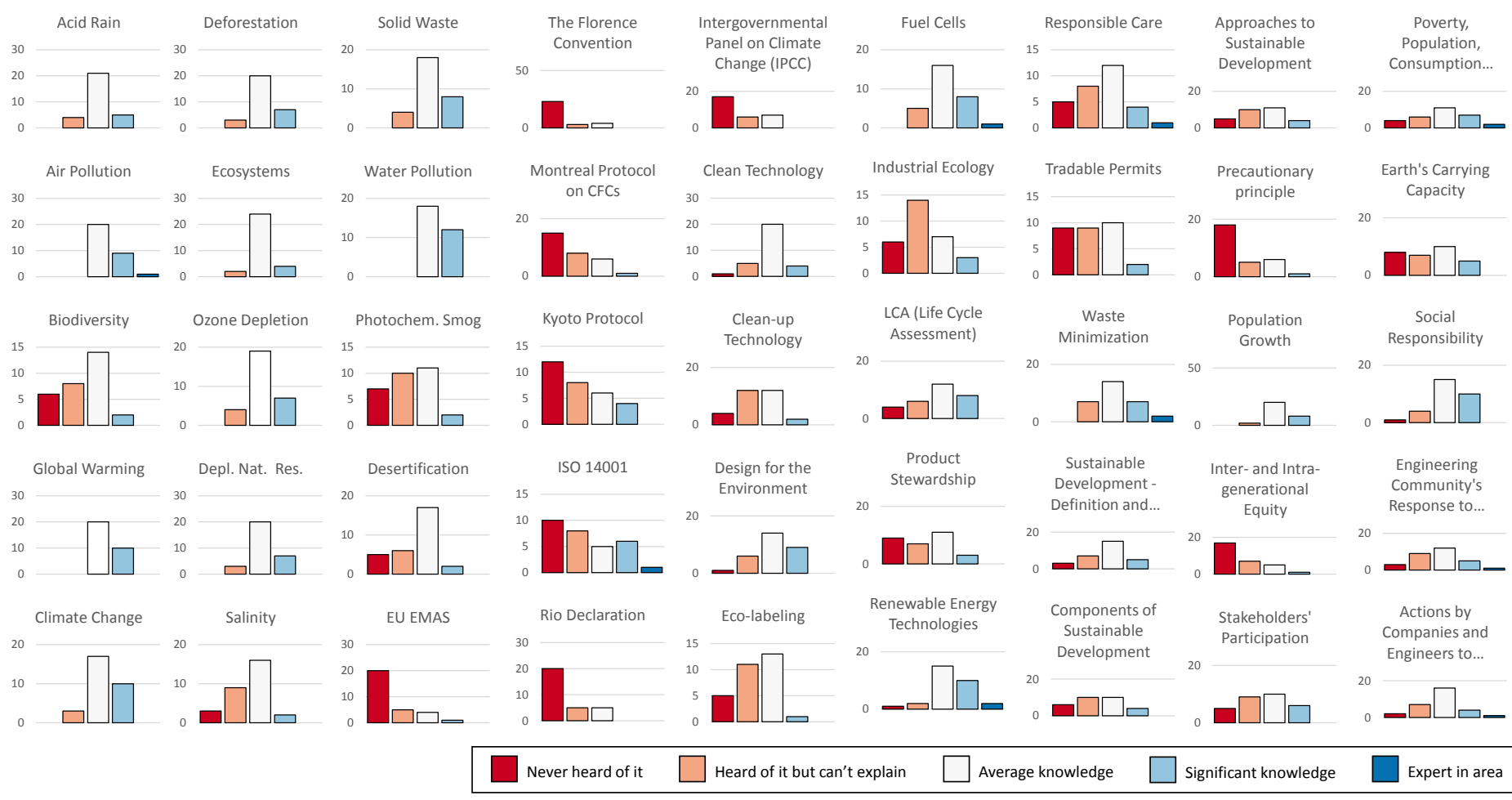

FIGURE 1. Summary of results from the preliminary user survey (total of 28 respondents). Each barchart shows responses for self-perceived awareness in that particular topic. Topics chosen for this survey are based on a previous survey conducted in [2]. In general, participants had a low level of understanding related to eco-design and sustainability principles. Also, concepts that are popularized by media (climate change, global warming, corporate social responsibility) or have a direct bearing on engineering design (waste minimization, renewable energy) outperformed other categories.

concepts using such domain dependent design variables will allow better integration and usage of the former. Furthermore, we believe that using a design exploration based context for achieving this integration encourages critical thinking and promotes a deeper understanding of these concepts. Our methodology for contextualizing environmental sustainability to a particular engineering domain comprises of the following steps:

- Identification of Design Variables: Within any engineering domain, identify design variables that are commonly used for problem based learning. Among them, identify the relation between these variables to environmental performance. In theory, almost all variables will affect the environmental footprint of the resulting design. To simplify this process, it is best to select variables which have first-order (direct) correspondences

- Design Space Exploration: Construct a design exploration problem that requires the selection/tuning of variables to meet domain dependent design requirements. Along with these requirements, introduce an additional constraint of reducing the environmental impact of the design. The problem should be designed so that it requires careful consideration of the variables to reach an optimal solution. This can be achieved by setting up conflicting objectives or designing the problem to contain optimal solutions that violate rules of thumb

- Anchoring the Solution: Provide students access to domain experts and technical resources related to environmental sustainability and impact assessment. This will allow reflection on wrongly formed insights and developing a better understanding of relationships between sustainability performance and the involved design variables

- Motivating the Exploration Process: Provide incentives for students to challenge themselves in the exploration process and generate non-conventional solutions. A healthy competition can be fostered by means of providing grade incentives, performance based monetary rewards or social motivation through gamification of the problem

- Observing User Behavior: Explicitly record mistakes as well as new insights gained by the students. Understanding decision rationale is critical for breaking existing student mindsets and motivating the case for sustainable design 


\section{USER STUDY}

This section discusses a user study which was conducted to test the validity of our hypotheses. The primary objective of this study was to use a manageable user population make detailed observations (on a casewise basis) of user behavior to gain deeper insights into our integration framework. Engineering design is a diverse field that makes use of several related disciplines. In order to avoid scope creep, the current study was focused towards integrating environmental sustainability concepts within shape synthesis for machine design. Although results from the conducted study cannot be directly applied to all possible engineering disciplines, this simplification allows us to focus on understanding the learning effects of our framework. Results from the user study will be used to fine tune our methods for future studies in related engineering domains. To analyze the effect of incorporation sustainability concepts into a traditional design problem, we conducted a pilot study with a mechanical design expert with more than 10 years of industry experience. Feedback from this pilot study was used to refine the design tasks for a follow-up user study with 12 student engineers. Details of the conducted user study are discussed below.

\subsection{Background}

Structural synthesis represents a challenging design problem as it can include a wide range of subjective as well as quantifiable goals [21]. From a geometric standpoint, it requires the selection of a suitably sized member with an appropriate topology. Additional constraints, such as weight, stress and strain limits, allowable materials and manufacturing processes add complexity to this problem by limiting the design space. Rules of thumb and guidelines for synthesizing machine parts are well established in engineering literature [22] and are a part of existing undergraduate curriculum [23]. Conventionally, the goals for synthesis involve, 1) inducing a uniform load distribution over as much of the body as possible and 2) minimizing the weight or volume of the material consistent with cost and manufacturing processes. Based on the specified loading criterion, students learn generic principles and optimal seed shapes for synthesizing structural members. Designers' in the industry often use such principles to guide the synthesis process. Analysis tools such as finite element methods (FEA), are used as a means for validating and/or refining synthesized designs.

Papalambros (1990) [24] conducted a study that looked at the processes used by students' for synthesizing the shape of a structural member. In this study, student teams were required to design a bracket to transmit a specified force. Constraints for the design problem included, ease of manufacture, ability to carry the load without failure and weight reduction. The authors' observed that students' mostly used intuition and some amounts of low fidelity prototyping and FEA for designing the bracket. Thus, structural synthesis presents itself as a suitably complex exploration framework for enabling discovery learning. Em- bedding sustainability concepts in the "synthesis problem" can potentially allow students' to explore the relationships between environmental impact and domain specific design variables. It is our hope that this exploration process will allow students' to develop deeper insights regarding environmental sustainability. Such learning can be valuable for transitioning sustainability from an afterthought to an integral part of the design process.

The following sub-sections detail the experimental setup and the involved design tasks for our user study.

\subsection{Apparatus and Software}

We conducted our study on a Desktop PC with dual display screens. Participants used PTC Creo Paramteric 2.0 for constructing computer-aided-design (CAD) models of their design. For conducting finite element analysis (FEA) on the designs, the prescribed loads and constraints were applied using PTC Creo Simulate 2.0. The same software was also used for generating a tetrahedral parabolic mesh of the designs. ANSYS 14.0 was used for solving the loading condition as well as visualizing equivalent Von Mises stress. To simplify the calculation process of cradle-gate impact (computed using Eqn.1) an automatic calculator written in Microsoft Excel ${ }^{\circledR}$ VBA was provided to users. Users were also allowed to use a notebook for sketching out design and performing hand calculations. For this study, we did not permit users to look up relevant information from online or textual sources. However, each subject was closely monitored by a proctor with extensive experience in sustainable design and use of the involved software. When required, the proctor assisted subjects in using the involved software. We ensured that the proctor refrained from providing any form of conceptual or design related suggestions to users.

\subsection{Participants}

We recruited 12 paid participants (10 male, 2 female), aged between 18 and 30 years. Among them, 5 participants were in the graduate program and the rest ( 6 seniors, 1 junior) were in the undergraduate program within the School of Mechanical Engineering. Since our design tasks made use of specific engineering software (PTC Creo 2.0 and ANSYS), we ensured that participants were proficient in using them. All users were given a fixed remuneration for their participation in the design task. A list detailing the final weight and the single score of the top three performers was prominently displayed in the study area so that users could gauge their current level of performance. These measures allowed us to make the design tasks more competitive.

\subsection{Tasks}

For analyzing the effects of introducing sustainability related learning in the context of shape synthesis, users were required to complete two separate design tasks. In both tasks, the primary objective was to design a cantilever to be used in an automobile for a specified loading condition. The loading con- 


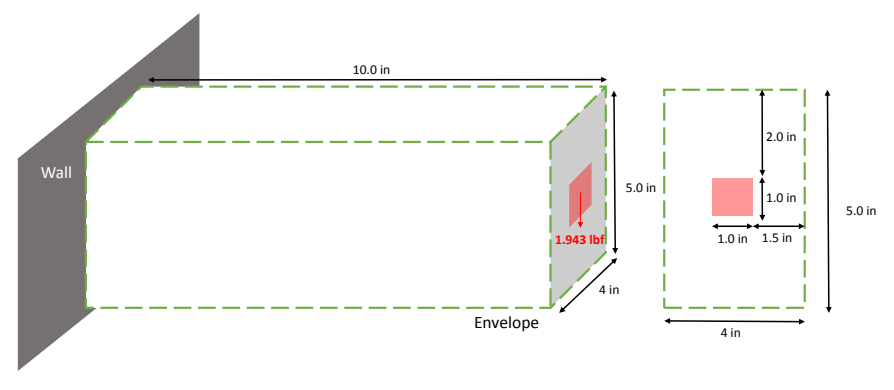

FIGURE 2. Figure illustrating the loading condition for both the design tasks in the user study. The box shown in dashed green represents the bounding envelope $(10 \mathrm{in} \times 5 \mathrm{in} \times 4 \mathrm{in})$ for the design. The square filled in red represents the area of application of the total load of $1.943 \mathrm{lbf}$.

dition (common to both tasks) is illustrated in Fig.2. There was no set number of design iterations that were specified. However, a total of twenty minutes was alloted for each design task. The differences between the two tasks stemmed from the design parameters that were required to be optimized.

- DESIGN TASK 1 (DT1) - Design task 1 was set up to familiarize users with the exploration framework and use of involved software. In DT1, users were required to minimize the total weight (and thus the volume) of the cantilever member such that it satisfied the specified set of constraints (C1-C4). The primary design constraint involving maximum allowable stress (C2) is purely a function of material geometry which means that a weight optimal solution will require a geometry that has a uniform distribution of stress close to the upper limit. Thus, DT1 enables users to iteratively explore several designs and understand the implicit relationships between shape, stress and weight

- DESIGN TASK 2 (DT2)- This task was setup to present a conflicting case between weight minimization (similar to DT1) and cradle-gate environmental impact of the designed member. In DT2, users were asked to select from three material alternatives: Cast Iron (GGL-NiCuCr), Aluminum (Al 2036) and Medium Carbon Steel (35S20). Each material had different values for the involved physical variables i.e. density, Young's modulus, maximum stress and environmental impact. Performing a complete life-cycle assessment (LCA) was outside the scope of this study. Therefore, a streamlined assessment was performed which involved the following stages:

- Material Extraction: The environmental impact associated with this life-cycle stage is given by the product of the weight of the initial blank and the impact associated with producing a blank of that material with unit weight

- Manufacturing: The design task only allows operations that remove material from the blank (C4). Therefore, any removal operation was treated to be a machining operation and its impact was calculated by multiplying the weight of material removed by the operation with the impact of the unit process associated with machining that material

- Material Recovery from Manufacturing: In addition to the impact associated with manufacturing, it was assumed that one hundred percent of the machined volume was recycled. Therefore an "environmental credit" equal to the weight of the machined volume multiplied with the impact of producing a blank of that material with unit weight was provided

Eqn.1 provides a mathematical representation of the overall cradle-gate environmental impact of the structural member.

$$
E I=k_{1} * W_{b}+k_{2} * \sum_{i=0}^{n} M R W_{i}-k_{3} * \sum_{i=0}^{n} M R W_{i}
$$

$\rightarrow W_{b}$ : weight of the starting blank

$\rightarrow M R W_{i}$ : weight of material removed in the $i^{\text {th }}$ manf. step

$\rightarrow n$ : total number of manufacturing steps

$\rightarrow k_{1}, k_{2}, k_{3}$ : Material/manufacturing process specific unit impacts calculated using the Ecoinvent 99(I) method on SimaPro®

The design constraints for DT1 and DT2 were also made to be the same so that users could translate their learning from the first task to the next. The specified constraints are listed below:

- CONSTRAINT 1 (C1): The design was required to lie within a bounding box measuring 10 in $\times$ in $\times 4$ in

- CONSTRAINT 2 (C2): The allowable equivalent Von Mises stress was less than half the value of "maximum stress" specified for each of the three materials

- CONSTRAINT $3(\mathbf{C 3})$ : In order to simply the design problem, users were required to start by selecting one of three crosssection shapes; Circular, rectangular of I-shaped

- CONSTRAINT 4 (C4): To maintain realism, only material removal operations were allowed. Therefore, in terms of modeling, users had to start with a blank and perform either extrude, revolve, sweep or blend cuts to realize the final shape. There was no restriction in terms of the number of CAD features or dimensions in the model

To measure the outcomes of the user study, a "think-aloud" protocol was used wherein participants were asked to vocalize their thoughts, observations and their approach for generating a solution. We also probed participants with questions relating to any significant observations that we made during the user study. An audio recorder was setup to capture this data and we also made extensive observation notes for every session. Our intent was to conduct a post-hoc analysis for understanding heuristics used to generate solutions, user conception of sustainability related topics and the effects of integrating sustainability based variables in a shape synthesis setting. An online survey related to possible learning outcomes, comments regarding the study and the taskload was administered at the end of DT2. Observations made 

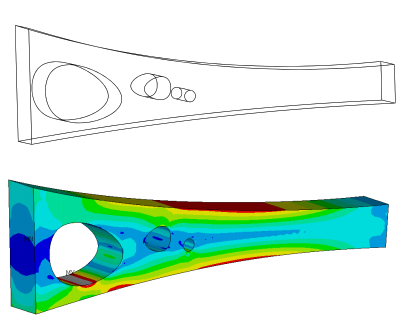

\section{Material Aluminum Weight $\quad 1.27 \mathrm{lbs}$ Impact $\quad 5.21 \mathrm{Pt}$}

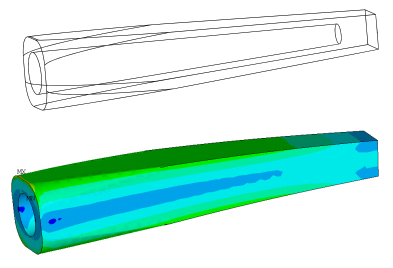

Material Aluminum Weight $1.69 \mathrm{lbs}$ Impact $6.93 \mathrm{Pt}$
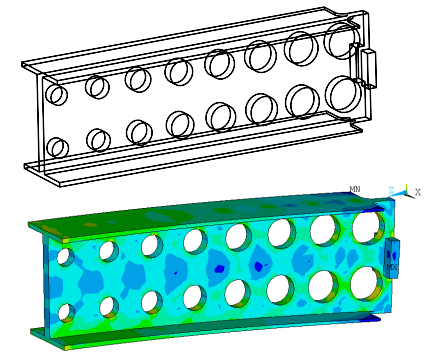

Aluminum

$1.01 \mathrm{lbs}$

5.52 Pt
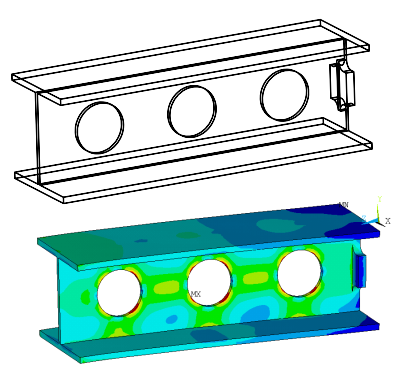

Aluminum $1.41 \mathrm{lbs}$ 6.64 Pt

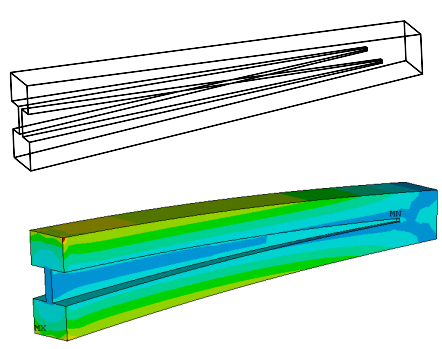

Aluminum

$1.36 \mathrm{lbs}$

$3.85 \mathrm{Pt}$

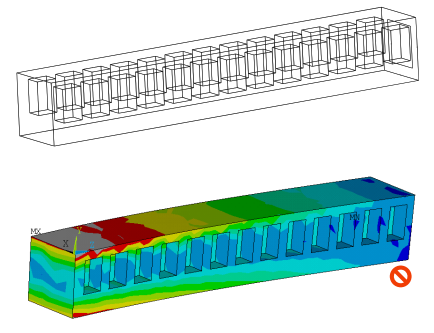

Cast Iron

$4.58 \mathrm{lbs}$

$6.9 \mathrm{Pt}$

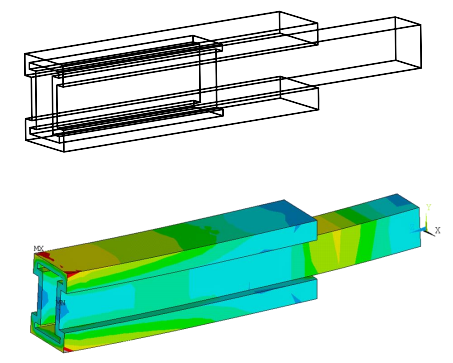

Carbon Steel

$4.24 \mathrm{lbs}$

3.63 Pt

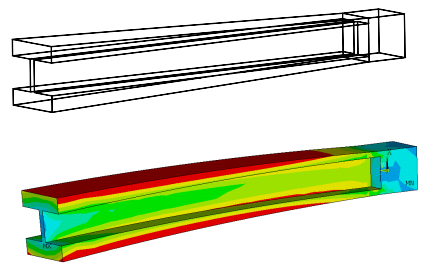

Carbon Steel

$2.21 \mathrm{lbs}$

5.14 Pt

Failed maximum stress limit constraint

FIGURE 3. Figure illustrating selected results from design task 2. The contour plot below each design shows the equivalent Von Mises stress (SEQV) calculated using FEA. The magnitude of SEQV is represented in psi and corresponding gradations are detailed in the color-scale located bottom right. As seen, designs based on Aluminum tend to be lighter, but environmental impact reduction is easier in Carbon Steel designs. A majority of users chose Aluminum blanks but only one of them was able to reduce the impact below $4 \mathrm{Pt}$ (Ecopoints).

from the audio recordings and notes were cross-checked with user comments from the survey to confirm our hypotheses. The results of the design tasks and the survey are detailed below.

\section{RESULTS}

The main goal of DT1 was to familiarize users with the workflow and have them draw insights on the implicit relationships between shape synthesis and induced stress. All 12 users had previously taken a course that taught design principles for static loading. Among them, 9 users had taken a computer-aideddesign course that also dealt with finite element analysis. Given the background of the participants, it is surprising to note that only half of them had been exposed to a design problem similar to DT1. We observed that DT1 enabled such users to develop a richer understanding of the involved concepts. One participant reported that "It was good to visualize the effect of variation in dimensions on the stress. The visualization of stress on the designed part helped on further refinement of the design by eliminating the low stress materials.”. Other users, who had previous exposure to similar problems reported that this task helped validate design insights that they had developed from previous mechanics courses. For example, one participant said "The task confirmed my insight the material should be located as far away from the neutral axis to reduce the amount of material required. The system can be reduced to a shear force at the attachment points and a bending moment on the beam." Feedback from the online survey also indicate that tasks similar to DT1 offer new insights that students miss within traditional mechanical engineering courses. Users commented that:

- "It was interesting to use finite element analysis (FEA) for a real problem as being able to see where maximum stress occurs is very helpful in learning where to take out more material from"

- "The selection of base shape material is important to enhance the chance of shape change in the design process. For example if I select circle, one way I can imagine to change the material shape is changing radius. However, the I-shape could provide more possibilities in the change of shapes (changing each dimension of sides)" 
From the think-aloud data we observed that in several cases, the design task helped disprove false intuitions and mental models formed by student engineers. The exploratory nature of the task allowed students to critically examine previously learnt concepts and forced them to make sense of unexpected results. As one participant said; "Sometimes intuition and experience is not enough to know exactly where high stress will occur with complex geometry and FEA is a useful tool in those cases."

For DT2, users were asked to build on insights from DT1 and simultaneously consider the effects of the cradle-gate impact of their designs. A snapshot of selected user solutions for DT2 is illustrated in Fig.3. From a total of twelve designs, two of them failed to meet the criterion for limiting the maximum value of equivalent Von Mises Stress. Analysis of data from the study shows that users primarily relied on past experience for initially selecting a material type. For example, a majority of users (7 of 12) preferred Aluminum over Cast Iron and Medium Carbon Steel because of their intuition that they could make the design very light as well as use lesser material in the process. Although, this intuition worked for a few designs, students immediately realized that this selection significantly constrained the design space. A rectangular shaped blank was preferred in DT2 as most users ( 7 of 12) felt that it offered more flexibility in terms of material removal operations. The I-shaped blank was chosen by three users who reasoned that the shape offers much more stiffness in bending when compared to the other shapes. All 12 results for DT2 are detailed in Fig.4.

Similar to the results from the preliminary user survey, the online survey shows that the entire user group felt that learning concepts about sustainable design was important for design engineering. However, only 2 participants had previous training in concepts related sustainability and 1 participant (with no previous training) was considering taking related coursework. Previous surveys we have conducted have also shown similar results supporting the hypothesis that current engineering curriculum does not motivate students to enroll in a separate course devoted to sustainability. This issue is compounded by the fact that most sustainability courses that teach life-cycle and systems engineering related concepts fail to link them to common design practice. Our analysis of the think-aloud data shows that inclusion of exploratory design tasks that contextualize sustainability within existing curriculum can overcome these shortcomings. User feedback on DT2 strongly supports this view as all 12 users felt that the design task was within the context of current engineering mechanics curricula. The think-aloud data shows several instances where users' formed new insights relating sustainability and shape synthesis. User comments on DT2 support this observation. They point out that:

- "It was interesting to see how certain materials are better for certain types of problems; Aluminum is expensive to cast but relatively cheap to machine down after the casting has been done"

\begin{tabular}{|c|c|c|c|c|c|c|c|}
\hline Material & Blank C.S & $\begin{array}{c}\text { Weight } \\
\text { (lbs) }\end{array}$ & $\begin{array}{c}\text { Single } \\
\text { Score } \\
(\mathrm{Pt})\end{array}$ & $\begin{array}{c}\text { Blank } \\
\text { Volume } \\
(\text { in^3) }\end{array}$ & $\begin{array}{c}\text { Final } \\
\text { Volume } \\
\left(\text { in`3) }^{\circ}\right.\end{array}$ & $\begin{array}{c}\text { Weight } \\
\text { (lbs) }\end{array}$ & $\begin{array}{c}\text { Single } \\
\text { Score }(\mathrm{Pt})\end{array}$ \\
\hline \multirow{8}{*}{ Aluminum } & & 2.08 & 4.49 & 24.00 & 20.92 & & \\
\hline & I-shape & 1.01 & 5.52 & 32.50 & 10.18 & & \\
\hline & & 1.42 & 6.65 & 38.75 & 14.25 & & \\
\hline & & 1.36 & 3.85 & 21.40 & 13.71 & & \\
\hline & & 1.21 & 5.18 & 30.00 & 12.16 & & \\
\hline & Rect. & 1.27 & 5.20 & 30.00 & 12.78 & & \\
\hline & & 1.69 & 6.93 & 40.00 & 17.00 & & \\
\hline & & 2.15 & 7.08 & 40.00 & 21.62 & & \\
\hline \multirow{3}{*}{ Carbon Steel } & I-shape & 4.24 & 3.63 & 25.00 & 14.99 & & \\
\hline & Rect & 3.05 & 3.31 & 20.00 & 10.78 & & \\
\hline & Nect. & 2.21 & 5.14 & 22.50 & 7.82 & & \\
\hline Cast Iron & Rect. & 4.59 & 6.90 & 30.00 & 18.34 & & \\
\hline
\end{tabular}

FIGURE 4. Table detailing results from all the twelve user studies. In this figure "material" and "blank cross-section" are attributes defined for the user chosen blank within DT2. "Weight" and "single score" (cradlegate as per Eq.1) are calculated with respect to the final design in DT2. "Blank volume" and "final volume" are also calculated based on user responses within DT2. The last two columns of the table provide a visual overview of the numerical values of "weight" and "single score". Values highlighted in red represent designs that failed the maximum allowable stress constraint $(\mathrm{C} 2)$.

- "I tend to forget that the scrap material can be recycled. When I approach design I try to minimize the amount of scrap"

- "This task also caused one to balance carrying the load along with not wasting material, which really changes the thought process for designing"

- "Normally, we tend to only focus on minimizing volume when considering sustainable design. Rarely do we think about how much energy is required to minimize that volume. We come up with fancy and intricate designs to reduce volume, but neglect the manuf. effects associated with this process. In most undergrad courses, this is not even mentioned as an important factor"

Furthermore, results from the online survey show that 11 users out of the 12 agreed that DT2 could be easily integrated into existing mechanics courses that they had previously taken. Additionally, a total of 7 users reported that this exercise convinced them to take a deeper look into sustainable design concepts. Results also show that the average likelihood for participants to use sustainability as one of the guiding principles in future designs was equal to 4.08 (on a linear 1-5 scale).

\section{DISCUSSION}

Based on the insights developed from the preliminary user survey and our shape synthesis user study, we try and answer questions that we put forward in the beginning of this paper. 


\subsection{Takeaways}

Q1: What are the knowledge gaps in sustainable design present in mechanical engineering students?

A1: Results from our preliminary survey show that two kinds of knowledge gaps exist; A lack of awareness of sustainability related concepts and inability to contextualize these concepts into common design practice. These gaps are not addressed within current engineering curriculum as 1) most students do not enroll in a class that is devoted to sustainable design and 2) often, such classes lack relevance to mechanical engineering domains. While the aim of this paper was to investigate existing sustainability related knowledge gaps and develop methods for curriculum integration, our user studies have provided us with a broader understanding of the problem. Even though our user study was limited to twelve students, we observed that a worryingly large number of users failed to use previously learnt insights from related domains (i.e. mechanics, vehicle design and material science) to guide their designs. A potential explanation for these observations is the lack of exposure to multi-domain design problems until much later (junior or senior years) within existing curricula. Providing a real-world design context to traditional mechanical engineering courses could help break these isolated silos of knowledge. Another concern is the lack of engineering tools that can support design exploration without significant cognitive overheads. In most cases, students are forced to either explore solutions by hand or spend significant time in learning design-and-analysis software before they can ask "what if?" questions. These same observations hold for sustainable design teaching wherein the context of "multi-domain experience" and "lack of supporting software" become rather apparent.

Q2: How can we integrate sustainability based learning into design engineering curricula to address some of these gaps?

A2: In section 2, we have reviewed existing literature on sustainability based learning. These efforts have been successful in galvanizing universities to include new courses, programs and departments focusing on sustainability and environmental engineering. We believe that the next step in sustainability education is to ensure that every engineering graduate is equipped with the learning and tools to address this issue. As pointed out earlier, the current setting falls short of these goals due to the fact that only a handful of courses attempt to integrate sustainability into common design practice. Results from our user study have shown that contextualizing sustainability concepts by framing them in a design exploration context can significantly alleviate such gaps. Adopting a framework similar to ours, also allows students to gain new insights and reflect on the implicit relationships between design parameters and sustainability related variables. User feedback has also shown promise in terms of students applying this learning towards future design projects. In summary, we conclude that engineering curricula should 1) consist of modules within traditional courses that contextualize sustain- ability within that particular domain, 2) adopt a design exploration based framework that exposes students to multi-domain problems and 3) continue developing (more advanced) courses devoted to teaching a life-cycle and systems level perspective on these concepts. Finally, it is important that these modules be introduced at an early stage, when students are developing insights related to fundamental engineering principles.

\subsection{Summary and Future Work}

This paper has detailed current knowledge gaps present in mechanical engineering students from a sustainability perspective. Based on results from our preliminary survey of graduate engineers, we develop a framework for contextualizing sustainability within engineering design curricula. To validate our framework, we conducted a user study that used shape synthesis as the design context. Results from the user study have underscored the importance of contextualizing sustainability teaching to specific engineering domains. Our observations of users and their feedback about the design tasks have helped us establish goals for related future efforts. We conclude our discussion by listing out important limitations present in the current study means for overcoming them.

- Our user study was limited to a participant pool of twelve as our primary objective was to make detailed observations of user behavior to formulate the bases for a subsequent summative study. A larger participant pool would have prevented us from using a think-aloud protocol which was instrumental in gain deeper insights related to this problem. We are planning on an extended version of this study which will be conducted in a classroom environment. We are also looking at the possibility of conducting similar studies within engineering courses teaching heat transfer and fluid mechanics

- Feedback from our user study also indicated the design constraints such as the initial shape of the blank and time for completion prevented some students from generating a more optimal solution. Although it is arguable that similar constraints are faced by mechanical engineers in the real world, we would like to conduct a similar study that relaxes these constraints

- In this study, we were limited to a participant pool that was proficient in specific engineering software. Future studies, will look at developing a software platform that has lesser training barriers. A possible solution could involve the use of natural user interface based software platforms for CAD and FEA

- Finally, to ensure applicability of learnt concepts, our integration framework for sustainability should enable change at a "conceptual level" among users. When presenting information inconsistent with existing conceptual structures, students tend to develop misconceptions regarding these concepts [25]. Future work will investigate methods that can ease this transition 


\section{ACKNOWLEDGMENTS}

Thanks to to Dr. Tahira Reid, Joran Booth and Senthil Chandrasegaran for contributing towards the user study and providing feedback on the document. This research is partially supported by NSF under grants CMMI 110619 and DUE 245780. The contents of this manuscript do not necessarily reflect the views or opinions of the funding agency.

\section{REFERENCES}

[1] Accreditation Board for Engineering \& Technology, 2013. Accreditation criteria and the accreditation policy and procedure manual.

http://www.abet.org/accreditation-criteria-policies-documents/. Accessed 2014-01-06.

[2] Azapagic, A., Perdan, S., and Shallcross, D., 2005. "How much do engineering students know about sustainable development? the findings of an international survey and possible implications for the engineering curriculum". European Journal of Engineering Education, 30(1), pp. 1-19.

[3] American Society of Mechanical Engineering, 2010. Sustainable design trend watch survey results.

http://memagazine.asme.org/web/Sustainable_Design_Trend.cfm. Accessed 2010-06-06.

[4] Tilbury, D., 1995. "Environmental education for sustainability: Defining the new focus of environmental education in the 1990s". Environmental Education Research, 1(2), pp. 195-212.

[5] Fien, J., and Tilbury, D., 2002. "The global challenge of sustainability". Education and sustainability: Responding to the global challenge, pp. 1-13.

[6] Ashford, N. A., 2004. "Major challenges to engineering education for sustainable development: what has to change to make it creative, effective, and acceptable to the established disciplines?". International Journal of Sustainability in Higher Education, 5(3), pp. 239-250.

[7] Brundiers, K., Wiek, A., and Redman, C. L., 2010. "Real-world learning opportunities in sustainability: from classroom into the real world". International Journal of Sustainability in Higher Education, 11(4), pp. 308-324.

[8] Dieleman, H., and Huisingh, D., 2006. "Games by which to learn and teach about sustainable development: exploring the relevance of games and experiential learning for sustainability". Journal of Cleaner Production, 14(9), pp. 837-847.

[9] Antle, A. N., Bevans, A., Tanenbaum, J., Seaborn, K., and Wang, S., 2011. "Futura: design for collaborative learning and game play on a multi-touch digital tabletop". In Proceedings of the fifth international conference on Tangible, embedded, and embodied interaction, ACM, pp. 93-100.

[10] Brewer, R. S., Lee, G. E., Xu, Y., Desiato, C., Katchuck, M., and Johnson, P. M., 2011. "Lights off. game on. the kukui cup: A dorm energy competition". In Proceedings of the CHI 2011 Workshop Gamification: Using Game Design Elements in
Non-Game Contexts.

[11] Gennett, Z. A., Isaacs, J. A., and Seager, T. P., 2010. "Developing a social capital metric for use in an educational computer game". In Sustainable Systems and Technology (ISSST), 2010 IEEE International Symposium on, IEEE, pp. 1-6.

[12] Katsaliaki, K., and Mustafee, N., 2012. "A survey of serious games on sustainable development". In Simulation Conference (WSC), Proceedings of the 2012 Winter, IEEE, pp. 1-13.

[13] Steinemann, A., 2003. "Implementing sustainable development through problem-based learning: Pedagogy and practice". Journal of Professional Issues in Engineering Education and Practice, 129(4), pp. 216-224.

[14] Ameta, G., Panchal, J. H., and Pezeshki, C., 2010. "A collective-learning approach to sustainable design education". International Journal of Engineering Education, 26(2), p. 265.

[15] Bernstein, W. Z., Ramanujan, D., Zhao, F., Ramani, K., and Cox, M. F., 2012. "Teaching design for environment through critique within a project-based product design course". International Journal of Engineering Education, 28(4), pp. 1-12.

[16] Peet, D.-J., Mulder, K., and Bijma, A., 2004. "Integrating sd into engineering courses at the delft university of technology: The individual interaction method". International Journal of Sustainability in Higher Education, 5(3), pp. 278-288.

[17] Kumar, V., Haapala, K. R., Rivera, J. L., Hutchins, M. J., Endres, W. J., Gershenson, J. K., Michalek, D. J., and Sutherland, J. W., 2005. "Infusing sustainability principles into manufacturing/mechanical engineering curricula". Journal of manufacturing systems, 24(3), pp. 215-225.

[18] Warburton, K., 2003. "Deep learning and education for sustainability". International Journal of Sustainability in Higher Education, 4(1), pp. 44-56.

[19] Shephard, K., 2008. "Higher education for sustainability: seeking affective learning outcomes". International Journal of Sustainability in Higher Education, 9(1), pp. 87-98.

[20] Entwistle, N., 2000. "Promoting deep learning through teaching and assessment: conceptual frameworks and educational contexts". In TLRP conference, Leicester.

[21] Papalambros, P. Y., and Shea, K., 2001. "Creating structural configurations". In Formal engineering design synthesis, E. K. Antonsson and J. Cagan, eds. Cambridge University Press.

[22] Weaver, P., and Ashby, M., 1996. "The optimal selection of material and section-shape". Journal of Engeering Design, 7(2), pp. 129-150.

[23] Starkey, J. M., and Starkey, W. L., 2010. Shape synthesis of high-performance machine parts and joints. Lecture notes ME 455: Vehicle Design and Fabrication.

[24] Papalambros, P. Y., and Chirehdast, M., 1990. "An integrated environment for structural configuration design". Journal of Engineering Design, 1(1), pp. 73-96.

[25] Vosniadou, S., 1994. "Capturing and modeling the process of conceptual change". Learning and instruction, 4(1), pp. 45-69. 